\title{
Regional delivery of immunosuppression for transplantation of vascularized composite allografts: opportunities near and far
}

\author{
Jeffrey L. Platt ${ }^{1}$, Marilia Cascalho ${ }^{1}$, Christina L. Kaufman ${ }^{2}$ \\ ${ }^{1}$ Department of Surgery and Department of Microbiology \& Immunology, University of Michigan, Ann Arbor, MI, USA; ${ }^{2}$ Department of \\ Cardiovascular and Thoracic Surgery and the Trager Transplant Center, University of Louisville, Louisville, KY, USA \\ Correspondence to: Jeffrey L. Platt, MD. Department of Surgery and Department of Microbiology \& Immunology, University of Michigan, \\ Transplantation Biology Program A520B MSRB I, 1150 W. Medical Center Drive, SPC 5656, Ann Arbor, MI 48109-5656, USA. \\ Email: plattjl@umich.edu. \\ Comment on: Lellouch AG, Taveau CB, Andrews AR, et al. Local FK506 implants in non-human primates to prevent early acute rejection in \\ vascularized composite allografts. Ann Transl Med 2021;9:1070.
}

Submitted Oct 06, 2021. Accepted for publication Oct 21, 2021.

doi: $10.21037 /$ atm-2021-15

View this article at: https://dx.doi.org/10.21037/atm-2021-15

The pathway to innovation of vascularized composite allografts (VCA), such as grafts of allogeneic limbs or face, differs in important ways from pathway to innovation of clinical organ allografts. Although innovations in organ transplantation might be tested initially in large animal models, which better represent some aspects of human biology than laboratory mice, ultimately innovations in organ transplantation must be optimized in carefully planned and well powered clinical trials before a decision is made about whether clinical application is warranted. Innovations in VCA transplantation might also undergo initial testing in large animal models, such as the model Lellouch et al. (1) used to explore the potential utility of FK506-impregnated disks in VCA transplantation, but ultimately decisions about application must be reached without relying on results of clinical trials like those now standard in organ transplantation. This difference between organ and VCA transplantation reflects the profound difference in experience-currently about 150,000 organ transplants are performed world-wide, while in the most prolific year 15 VCA were performed.

\section{Optimizing immunosuppression for VCA}

If recipients of VCA are scarce, the imperative for optimizing immunosuppression while minimizing risks is compelling. In contrast to organ transplants, VCA usually are not performed to rescue the recipient from a severe, potentially lethal condition but rather to correct disability and improve the quality of life. Nor are VCA considered "life-supporting" transplants, as are organ transplants. Consistent with these distinctions, the recipients of clinical VCA, such as limb or face transplants, are generally healthy and presence of a significant underlying disease might contraindicate VCA transplantation. Organ transplant recipients always have a serious underlying disease and a person with clinically stable and/or mild condition would be directed away from organ transplantation and would be among the last to be offered a donated organ. Because VCA recipients are healthy, the immunosuppressive regimens used to avert rejection should minimize the risk of life-threatening complications and toxicities. Indeed, the VCA graft and not the recipient should bear the greater burden of risk. In contrast, immunosuppressive regimens used in organ transplantation might and often do impose risks of serious complications because survival of the recipient depends on survival of the graft.

Lellouch and co-workers have long sought novel approaches to minimizing the risks of immunosuppressive therapy in VCA transplantation. As one approach, the authors have been exploring local delivery immunosuppression, reasoning that success would allow the dose and hence the risk of systemic immunosuppression to be decreased. As another approach the authors have explored the longstanding idea that induction and maintenance of allogeneic tolerance through the engrafting of allogeneic hematopoietic 
cells might eliminate the need for immunosuppressive therapy thereby eliminating risk. The current report (1) describes experiments in which film-like disks impregnated with FK506, a calcineurin inhibitor also known as tacrolimus, were implanted below skin edges of allogeneic facial VCA in cynomolgus monkeys in efforts to decrease the need for systemic FK506 delivery to support VCA. The authors sought to optimize dose and formulation and to test whether local delivery of FK506 in this way could avert rejection and enable development of chimerism. Local delivery of FK506 and other drugs was previously reported to prevent rejection of VCA in rodents, but experience in randomly bred large animals and in clinical settings is quite limited.

The experiments described in this new report show that this new approach does contribute to immunosuppression and in conjunction with low doses of other agents and FK506 given systemically may help thwart rejection of VCA. Since FK506 is approved for topical use in skin diseases, such as psoriasis, evidence of efficacy of local FK506 in non-human primates might be taken to support use in clinical VCA recipients. However, several monkeys in which FK506-impregnated film was introduced exhibited toxicity. One monkey developed unacceptably high levels of the drug, which the authors ascribe to flawed formulation, and three subsequently treated with whole body irradiation and allogeneic bone marrow experienced post-transplant lymphoproliferative disease. The question then is how one should weigh evidence of biological efficacy against the evidence of toxicity in experiments performed in a small number of non-human primates?

We are more impressed by evidence of biological efficacy. The implantation of impregnated film effectively delivers FK506 since rejection is indeed prevented. Moreover, the "toxicity" Lellouch et al. (1) describe largely reflects effective release of the drug leading to high, potentially toxic, systemic levels. Since the number and formulation of disks and the frequency of implantation remain to be optimized, the results support the possibility that optimum use of disks as local source of FK506 could allow the doses of oral FK506 to be decreased leading to a net decrease in risks imposed by the drug. However, the small number of monkeys used cannot be taken to represent the range of responses likely to be seen in a large diverse population of treated individuals.

On the other hand, we see little evidence of toxicity of FK506 unequivocally linked to local delivery. We do not consider monkeys conditioned for hematopoietic stem cell engraftment to represent a clinically relevant test of local FK506 for VCA recipients. Such conditioning is not a standard practice in VCA transplantation. Rather, occurrence of lymphoproliferative disease could be taken to reflect effective control of development and/or functions of effector memory $\mathrm{T}$ cells manifest as loss of T-dependent viral latency. Hence, while we commend the conservative interpretation Lellouch et al. (1) apply to their results we do not believe clear evidence of toxicity was observed under conditions that represent current management of VCA.

\section{Challenges and opportunities associated with large animal models and clinical trials in transplantation}

New immunosuppressive drugs or regimens in transplantation are nearly always tested in non-human primates. However, complex models using non-human primates often under-represent efficacy and over-represent complications and toxicity that would be observed in clinical settings when use has been optimized and risks ameliorated by availability of more complete and effective monitoring (2). Regardless of the weight one might give evidence of efficacy and toxicity reported by Lellouch et al. (1), the findings highlight one difference between VCA from organ transplantation that has the most profound implications for how innovations can be advanced to clinical application.

Although recipients of VCA and recipients of organ transplants receive similar amounts of immunosuppression (3), rejection occurs at higher frequency in clinical VCA than in clinical organ transplants. During the first year after transplantation nearly all VCA undergo a rejection reaction, some repeatedly, while only $10-15 \%$ of kidney transplants undergo a proven rejection reaction $(4,5)$. This difference makes it possible to test mitigation of rejection in clinically relevant conditions (e.g., with current regimens of immunosuppression) using relatively few non-human primates. Although statistical tests were not applicable for this preliminary examination local FK506, the authors claim that the considerable experience at their center indicates results of three transplants per condition in non-human primates would "provide statistical power for the detection of survival differences" (1). Regardless of one's views concerning the authors' claims, three animals do provide a power of $80 \%$ in a VCA model in which $90 \%$ of controls experienced rejection and treated animals did not. In contrast, more than 90 kidney 
transplants in non-human primates would be needed to achieve the same power for testing an innovation that would mitigate rejection. Indeed, the scarcity of acute rejection of organ transplantation has made it necessary to employ sub-optimal immunosuppression in experimental models and to use endpoints other than acute rejection in clinical trials. For example, clinical trials in kidney transplantation now commonly use long term (5 or 10 years) outcomes or surrogate markers of immunity in lieu of acute rejection (6) and experiments in non-human primates might include cessation or suboptimal dosing of immunosuppression to test innovations in immunosuppression. What deserves wider recognition then is that notwithstanding technical challenges, the high frequency of rejection, makes clinical and experimental VCA (in large animals given current regimens of immunosuppression) profoundly more efficient systems for testing innovations like FK506 implants.

\section{The high frequency of acute rejection in VCA}

Why does rejection occur with such greater frequently in clinical VCA than in clinical organ transplants? Some postulate the high frequency of rejection of VCA reflects the presence of allogeneic skin, which is more immunogenic than allogeneic organ tissue. We certainly agree that skin is quite immunogenic, skin allografts in unmodified recipients nearly always evoke alloantibody responses and can reliably distinguish monozygotic from di-zygotic relationships. However, organ allografts may be more immunogenic than commonly appreciated. Clinical renal allografts always generate donor-specific B cell responses (7) and like skin allografts always fail after transplantation into unmodified recipients (8). More important however is that other differences between VCA and organ transplants may suggest avenues for fundamental and clinical progress.

Another explanation for the high frequency of rejection in VCA reflects the ability to continuously visualize the skin component of VCA. Because skin is quite sensitive to regional inflammation and changes are quickly seen or felt by recipients even minor episodes of rejection are reported and potentially treated. In contrast, rejection of organ transplants sometimes must await periodic visits to clinics and monitoring of blood or surveillance biopsies. The ready availability of skin for inspection and biopsy allows nearly continuous assessment of interventions and potentially facilitates repeated treatment with local FK506.

\section{Local delivery of immunosuppression for cell- mediated conditions?}

The report of Lellouch et al. (1) might prompt yet another question and that is why local delivery of FK506 would be expected to exert any biological impact. Although FK506 is thought to prevent rejection of allografts by blocking activation of naïve and memory $\mathrm{T}$ cells, this mechanism does not fully explain the efficacy and limitations observed in clinical transplantation or the prospects for efficacy of local delivery. Thus, while FK506 appears to hinder some functions of stimulated naïve and memory $\mathrm{T}$ cells tested in vitro, experimental and clinical experience indicates the agent suppresses naïve $T$ cells more effectively than memory T cells (9). Thus, FK506 can prevent rejection of unsensitized recipients but by itself poorly if ever prevents rejection of sensitized recipients. Likewise, memory $\mathrm{T}$ cells in most transplant recipients receiving FK506 can exert at least some control of latency of ubiquitous human herpesviruses. But, since activation of all naïve $\mathrm{T}$ cells (and some memory $\mathrm{T}$ cells) occurs exclusively in lymphoid tissues efficacy of FK506 should depend on systemic levels that deliver the drug to lymphoid organs. If that is correct then the principal benefit of local delivery might reflect the relatively continuous elution of the drug from the implants, averting the fluctuation in drug levels associated with intermittent oral or parenteral administration.

But, eliminating fluctuation of drug levels cannot explain how topical FK506 controls autoimmune diseases of skin, presumably mediated by effector memory $\mathrm{T}$ cells, like the effector memory $\mathrm{T}$ cells that control viral replication and latency and resist control by calcineurin inhibitors. We would entertain an alternative explanation for the efficacy of FK506 in rejection and autoimmunity. The migration of effector $T$ cells into target tissues and actions therein depends in part on interactions with endothelial cells that further activate the $\mathrm{T}$ cells and enable migration into target tissues. The interactions between $\mathrm{T}$ cells and endothelial cells depend in part on activation of endothelial cells. Antibodies that block these interactions are generally too toxic for clinical use, as the antibodies prevent would block the contribution of phagocytes to host defense throughout the body. Local suppression of $\mathrm{T}$ cell-endothelial cell interaction would seem preferable. Some years ago we showed that FK506 blocks transcription of a select set of genes in endothelial cells, including genes encoding proteins that could enable interaction with effector memory $\mathrm{T}$ cells 
$(10,11)$. While systemic delivery of FK506 undoubtedly suppresses activation of naïve $\mathrm{T}$ cells and perhaps some memory T cells, local delivery of FK506 might confer benefit by a different mechanism. If FK506 indeed does inhibit interactions between effector $\mathrm{T}$ cells and endothelial cells, that could explain why calcineurin inhibitors so effectively prevent rejection reactions mediated by memory T cells and how local delivery of FK506 to small areas of skin effectively thwart $\mathrm{T}$ cell-mediated injury at sites or lesions where the drug is applied but not elsewhere. We think the work of Lellouch et al. (1) could offer insights of potential import in VCA transplantation and beyond.

\section{Acknowledgments}

Funding: Work of the authors pertinent to transplantation is supported by grants from the National Institutes of Health (AI151588) and the Department of Defense (W81XWH-20-1-0943).

\section{Footnote}

Provenance and Peer Review: This article was commissioned by the editorial office, Annals of Translational Medicine. The article did not undergo external peer review.

Conflicts of Interest: All authors have completed the ICMJE uniform disclosure form (available at http://dx.doi. org/10.21037/atm-2021-15). All authors report grant funding related to VCA (W81XWH-20-10943 DoD/ CDMRP). CLK has no personal financial conflicts but she is involved in review of VCA patients (VCA patient review) and in scientific and clinical societies related to VCA (Treasurer of Board, American Society of Reconstructive Transplantation). The authors have no other conflicts of interest to declare.

Ethical Statement: The authors are accountable for all aspects of the work in ensuring that questions related to the accuracy or integrity of any part of the work are appropriately investigated and resolved.

Open Access Statement: This is an Open Access article distributed in accordance with the Creative Commons Attribution-NonCommercial-NoDerivs 4.0 International License (CC BY-NC-ND 4.0), which permits the noncommercial replication and distribution of the article with the strict proviso that no changes or edits are made and the original work is properly cited (including links to both the formal publication through the relevant DOI and the license). See: https://creativecommons.org/ licenses/by-nc-nd/4.0/.

\section{References}

1. Lellouch AG, Taveau CB, Andrews AR, et al. Local FK506 implants in non-human primates to prevent early acute rejection in vascularized composite allografts. Ann Transl Med 2021;9:1070.

2. Platt JL, Piedrahita JA, Cascalho M. Clinical xenotransplantation of the heart: at the watershed. J Heart Lung Transplant 2020;39:758-60.

3. Rifkin WJ, Manjunath AK, Kantar RS, et al. A comparison of immunosuppression regimens in hand, face, and kidney transplantation. J Surg Res 2021;258:17-22.

4. Hein RE, Ruch DS, Klifto CS, et al. Hand transplantation in the United States: a review of the Organ Procurement and Transplantation Network/United Network for Organ Sharing Database. Am J Transplant 2020;20:1417-23.

5. Hariharan S, Israni AK, Danovitch G. Long-term survival after kidney transplantation. N Engl J Med 2021;385:729-43.

6. O'Connell PJ, Kuypers DR, Mannon RB, et al. Clinical trials for immunosuppression in transplantation: the case for reform and change in direction. Transplantation 2017;101:1527-34.

7. Lynch RJ, Silva IA, Chen BJ, et al. Cryptic B cell response to renal transplantation. Am J Transplant 2013;13:1713-23.

8. Hume DM, Merrill JP, Miller BF, et al. Experiences with renal homotransplantation in the human: report of nine cases. J Clin Invest 1955;34:327-82.

9. Benichou G, Gonzalez B, Marino J, et al. Role of memory $T$ cells in allograft rejection and tolerance. Front Immunol 2017;8:170.

10. Brunn GJ, Saadi S, Platt JL. Differential regulation of endothelial cell activation by complement and interleukin 1alpha. Circ Res 2006;98:793-800.

11. Brunn GJ, Saadi S, Platt JL. Constitutive repression of interleukin-1alpha in endothelial cells. Circ Res 2008;102:823-30.

Cite this article as: Platt JL, Cascalho M, Kaufman CL. Regional delivery of immunosuppression for transplantation of vascularized composite allografts: opportunities near and far. Ann Transl Med 2021;9(22):1635. doi: 10.21037/atm-2021-15 